\title{
特別講演
}

\section{Recent Progress in Rehabilitation Medicine}

\author{
Mathew H. M. Lee, M. D.*
}

The greying of America makes it necessary that we explore the concepts of geriatric rehabilitation and the epidemiology of disability in order to meet the health challenges of the United States for the 21st century. These issues will be discussed.

Future trends in pain rehabilitation, research from our Chronic Pain Laboratory, utilizing thermography as a diagnostic tool, and acupuncture as a therapeutic modality for pain abatement will be shared.

Since the beginning of time, the therapeutic effects of music on the general population have been utilized by various cultures. During the past decades, greater concern, interest and focus have been directed to the medical problems of the musician, the application of music therapy for the severely disabled, and the neurobiological study of the brain.

The advent of new tools to study the neuro- physiological effects of music and the passage of federal legislation have now converged to reestablish the historic bond between music and medicine.

The accomplishments of our music laboratory The Center for Research in the Arts and Medicine in the past decades and its future direction will be illustrated.

The aim of rehabilitation medicine is to restore a disabled person to maximum capacity -physical, emotional and vocational. Medical care cannot be considered complete until the patient with the residual disability has been trained "to live and work with what he has left."

To rehabilitation medicine the introduction of chronic pain management, music therapy, and the application of microcomputers adds new and vibrant dimensions ; and to patients, a holistic approach to care.

* Department of Rehabilitation Medicine, New York University Medical Center, U. S. A. 